\title{
A Localization and In-flight Alignment Protocol for Airborne SINS Based on Flight-vehicle Wireless Sensor Networks
}

\author{
Chao Gao* Jianhua Lu Guorong Zhao Shuang Pan \\ Department of Control Engineering \\ Naval Aeronautical and Astronautical University, Shandong 264001, P.R. China \\ * Corresponding author's Email: gaochao.shd@163.com
}

\begin{abstract}
The necessity recurrently comes to align a strapdown inertial navigation system (SINS) in a moving vehicle to guarantee the accuracy and efficiency in the long run-off of the inertial system after a take-off or launch command is issued. This in-flight alignment is therefore achieved by integrating SINS data with some external aiding source inlcluding airborne navigation equipments and networking sensors. In this paper, a localization architecture and alignment scheme is presented for aircraft in a three-demensional fleet network, which is based on wireless sensor network. Firstly, a 3D node localization scheme is designed based on weighed-multidimensional scaling, which adopt spherical locating in the initial stage, and adaptively choose source nodes with high relative reliability to achieve position update. Then a robust filter algorithm is applied to compensate time-varying delay error and large azimuth uncertainty in alignment. Extensive simulation shows that the DMDG-3D localization scheme can provide highly accurate and relatively reliable navigation information in real time, and $l_{2}-l_{\infty}$ filter algorithm can accelerate convergence and give better estimation of the navigation parameters.
\end{abstract}

Keywords: Wireless sensor network; 3D localization; Network-assistant; In-flight alignment; Robust filter

\section{Introduction}

The alignment of a strapdown inertial navagation system (SDINS) is the process whereby the orientation of body frame is determined with respect to the navigation frame[1]. In a broad sense, alignment of SDINS can be classified as initial alignment and in-flight alignment (IFA). The IFA is performed with IMU and external sensors during the vehicle flight[2,3], in which the primary assistant sources are satellite system, radar, or other airborne equipment, offering reference signals like position, velocity, or attitude to assist the alignment process[4,5]. However, these sources have disadvantages in redundancy, independence, stability in signal, which will be incipient faults in emergent take-off or warfare. So it is necessary to design a IFA scheme which can obtain navigation information from a integrative self-organizing system rather than a sort of information source.
With the progress of very-large-scale integration, micro-electro-mechanism system (MEMS) and wireless networking technology, the transition from flatform assistant to network assistant has attracted more attention lately. The envisioned applications of wireless sensor networks in military affairs range widely, such as "smart dust", target tracking, C4ISR et al. There are already some successful fleet networks, including joint tactical information distribution system (JTIDS), MIDS and so on. With the assistance of tactical data link (Link-16 or TTNT), the network can provide precise navigation information including position (or relative position), velocity, heading angle, and system clock. A fleet wireless sensor network is designed in the preliminary job with a integrated protocol architectures (DMDG) whose performance is certified by extensive simulation in literature $[6,7]$. The finial purpose of this paper is to put forward a localization and in-flight alignment algorithm for application layer which can propose high quality of 
precision and robustness with time-relay delay error and in-motion vehicles.

In the remainder of this paper, some previous work and evaluation indicator will first be derived in its system model. Then, a new 3D localization scheme will be presented. Following this, in-flight alignment utilizing a Fv-WSN interfusion will be realized based on $l_{2}-l_{\infty}$ robust filter. Finally, conclusions will be presented with the future work.

\section{Related work and system model}

Before presenting the localization scheme and alignment algorithm, we first give a brief account of important techniques about the Fv-WSN foundation.

Firstly, we specify the Fv-WSN adopting in this paper, which is detailed designed in the previous work [7], and summarized into the following points.

- Physical layer: high performance network terminal with send-receiver and preprocessor.

- Link layer: TTNT data link, anti-interference, available transfer radius for $50 \mathrm{~km}$.

- MAC/Routing: DMDG protocol (Ref.7), with TDMA/CSMA channel sharing mechanism, multi-hops, GLB-DMECR geographic routing.

Formally, the Fv-WSN is represented as a graph $G=\left(C, E, R_{c}\right)$, which consists of a set of mobile nodes, $C=\left\{C_{1}, C_{2}, \cdots, C_{m}\right\}$, a set of wireless links, $E$, and an adjustable transmission radius $R_{c}$. Especially, consider a vector device parameters $\gamma=\left[\gamma_{1}, \cdots, \gamma_{k+l}\right]$. Each device has one parameter. The unknown parameter vector is $\theta=\left[\theta_{1}, \cdots, \theta_{k}\right]$, where $\theta_{i}=\gamma_{i}$ $(i=1, \cdots, k)$. Note that $\left\{\gamma_{i}, i=k+1, \cdots, k+l\right\}$ are known.

\subsection{Evaluating indicators}

The following four components compose the evaluating indicator for localization and in-flight alignment performance, including coverage rate, link consumption indicator, time synchronism, and accuracy, in which the first two indicators have been defined in the previous research [6,7]. In this section, we will discuss the last two indicators[9-14].

- Time synchronism: mainly correlated with time-relay delay in transmission[8], which is estimated by send-receiver time, transfer time, and clock deviation et al. To meet the minimum requirements in range error $(\leq 10 \mathrm{~m})$, relative stability in frequency should less than $10^{-10}$, and alignment accuracy in frequency less than $5 \times 10^{-9}$, then the time-relay delay can meet the minimum base line $(\|\tau\| \leq 50 \mathrm{~ms})$.

- Accuracy: based on the post-processed relative position error $\Delta P_{\text {rela }}$, misalignment angle estimation $(\Delta \theta, \Delta y, \Delta \psi)$, and velocity error estimation $\left(\Delta V_{E}, \Delta V_{N}\right)$. It is assessed through mean, root mean square (RMS), minimum, or maximum computation.

\section{Three-dimensional localization scheme}

In this section, we specialize for location estimation using TOA measurement in a $\mathrm{Fv}-\mathrm{WSN}$. Specifically, consider $l$ reference and $k$ blindfolded nodes. The node parameters $X=\left[x_{1}, x_{2}, \cdots ; x_{k+1}\right]^{T}$, where, for 3D system, $x_{i}=\left[x_{i 1}, \cdots, x_{i j}\right], j=3$. The 3D localization problem corresponds to the estimation of blindfolded node coordinates $\theta=\left[\theta_{x}, \theta_{y}, \theta_{z}\right]$.

$$
\theta_{x}=\left[x_{1}, \cdots, x_{n}\right], \quad \theta_{y}=\left[y_{1}, \cdots, y_{n}\right], \quad \theta_{z}=\left[z_{1}, \cdots, z_{n}\right]
$$

\subsection{Localization scheme discription}

This subsection generally presents the main schedule of the localization scheme, which comprises four steps.

- Step 1: Informance gathering. Running in every initiation of its TDMA slot, gathered information involving available neighbor number $\left(d_{i j} \leq 2 \cdot r_{c}\right)$, 1-hop neighbor's detailed information $\quad\left(I D, P R L, T S_{C A}, C_{M s g} V_{C} \quad, \quad \phi_{C}, t_{A}\right.$, etc $)$ (defined in Ref.6), 2-hop relay information $\left(M T, C_{\operatorname{man}}\right)$, aggregate a set of neighbor nodes.

- Step 2: Geometric localization. Dynamically select four or more neighbors from the set (Step.1) based on PRI, GDOP, and distance $d_{i j}$, ranging by TOA, aggregate and update local coordinate $\left(x_{l o c} y_{l o c} z_{l o c}\right)$ by spheric localization algorithm, then establish or renew global relative coordinate. 
- Step 3: Stepwise refinement localization. Perform algorithm in paragraph 3.2, mainly to confirm adaptive weight vector $w_{i j}$, and obtain partial cost function $J_{i}$, which is used to update the global relative coordinate $\left(x_{\text {rela }} y_{\text {reld }} z_{\text {rela }}\right)$, and transform to global geodesic coordinate $\left(x_{a b s} y_{a b s} z_{a b s}\right)$ finally.

- Step 4: Upload information. Evaluate the result from Step. 3 and decide the cycle index, then broadcast the result by data link (forms in Ref.7).

\subsection{D localization algorithm}

As shown in step 2-3, vehicles first get four or more 2-hop neighbors and perform localization procedure based on Theorem.1, then adaptively choose source nodes with high reliability to achieve position update.

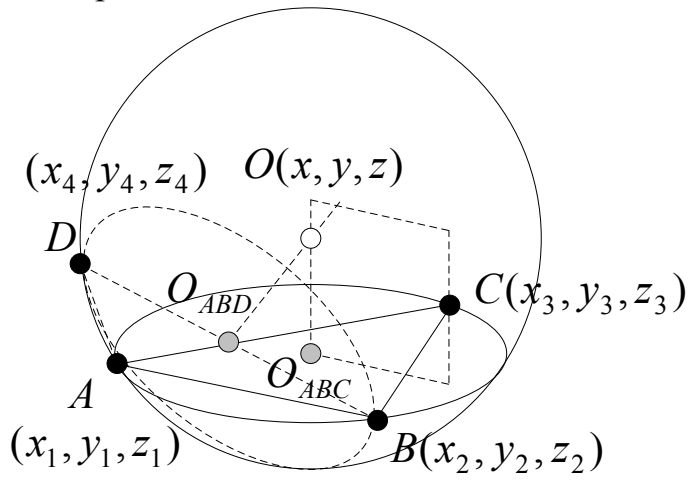

(a) Ideal distribution

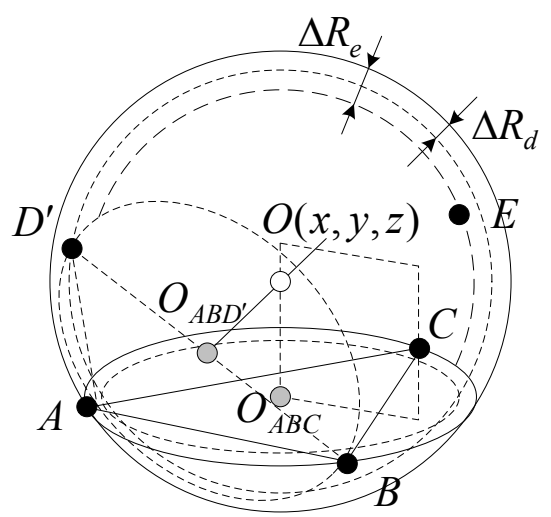

(b) Uncosphere distribution

Anchor node $\bigcirc$ Unknown node $\bigcirc$ Point of intersection

Figure 1. DMDG-3D localization scheme

Theorem 1. Given a sphere $O$, $O=\left\{(x, y, z)\left(x^{2}+y^{2}+z^{2}=r_{c}^{2}\right\}, \quad \exists A\left(x_{1}, y_{1}, z_{1}\right), B\left(x_{2}, y_{2}, z_{2}\right)\right.$, $C\left(x_{3}, y_{3}, z_{3}\right), \quad D\left(x_{4}, y_{4}, z_{4}\right) \in O$, if reference node $A, B, C, D$ is uncoplaner, as shown in Fig.1, the coordinate of sphere center $O(x, y, z)$ can be calculated uniquely $[6,15-19]$.

Theorem.1 is proven in Ref.6. Especially, if $A, B, C, D$ is uncosphere or collinear, as shown in Fig.1(b), the coordinate of $O(x, y, z)$ can't be confirmed directly. In this case, we adjust radius $r_{c}$ to select four or more optimal nodes within the same concentric sphere, e.g, in Fig.1(b), node $D$ is prior to $E$ because $\Delta R_{d}<\Delta R_{c}$, and the nodes $A, B, C, D^{\prime}$ are more approximate to a concentric sphere, then $(x, y, z)$ can be calculated by theorem. 1 , in which the positional errors in approximation are estimated by least-squares procedure.

Note that we realize the stepwise refinement localization by weighting least squares, as in SMA-COF algorithm[2], in which $\theta=\left[\theta_{x}, \theta_{y}, \theta_{z}\right]$ is the minimum optimum solution to partial cost function $J_{i}$.

$$
\begin{gathered}
J_{i}=\sum_{j=1, j \neq i}^{k} w_{i j}\left(\hat{d}_{i j}-d_{i j}(X)\right)^{2}+\sum_{j=k+1}^{k+l} w_{i j}\left(\hat{d}_{i j}-d_{i j}(X)\right)^{2} \\
+f\left(x_{i}\right)
\end{gathered}
$$

where $w_{i j}$ is weight vector, $w_{i j} \geq 0$. If there is no available measured value between node $i$ and $j$, $w_{i j}=0$. In (2), we substitude the distance value $\left|\hat{d}_{i j}^{2}-d_{i j}^{2}\right|$ into $\left|\hat{d}_{i j}-d_{i j}\right|$ to reduce the magnifying effect in weighting process.

Iterative routine is adopt to get the minimum solution for (2), in which the $(n+1)$ 's iterative result can be shown as in (3).

$$
x_{i}^{(n+1)}=\left[x^{(n)} g_{i}^{(n)}\right] /\left[\sum_{j=1, j \neq i}^{k} w_{i j}+2 \sum_{j=k+1}^{k+l} w_{i j}\right]
$$

where $g_{i}^{(n)}=\left[g_{1}, \cdots, g_{k+l}\right]^{T}$ is weight vector, which is defined as in (4) sectionally.

$$
g_{j}=\left\{\begin{array}{l}
w_{i j}\left[1-\hat{d}_{i j} / d_{i j}\left(X^{(n)}\right)\right], \quad j \leq k, j \neq i \\
2 \cdot w_{i j}\left[1-\hat{d}_{i j} / d_{i j}\left(X^{(n)}\right)\right], \quad j>k
\end{array}\right.
$$

The weighting mechanism is according with relative reliability $\lambda$, in which the relative reliability between node $i$ and $j$ can be calculated in (5).

$$
\lambda_{i}(j)=\left[\Delta R X_{i}(j)\right]^{-1}=\left[\Delta \bar{X}_{i}(j)+N X_{i}(i) \cdot \hat{d}_{i j}\right]^{-1}
$$

where $\Delta \bar{X}_{i}(j)$ is the self-localization error between 
node $i$ and 1-hop neighbor node $j . N X_{i}(i) \cdot \hat{d}_{i j}$ is the range error of node $i$ inducted by $\hat{d}_{i j}$. $\Delta R X_{i}(j)$ is the relative error considering $\Delta \bar{X}_{i}(j)$ and $N X_{i}(i)$, which can be calculated in (6-8) particularly.

$$
\begin{aligned}
& \Delta \bar{X}_{i}(j)=\frac{1}{m} \sum_{j=1}^{m}\left|\hat{d}_{i j}-d_{i j}\right| \quad j=1, \cdots, m \\
& N X_{i}(i)=\left[\sum_{j=1}^{m}\left|\hat{d}_{i j}-d_{i j}\right|\right] / \sum_{i=1}^{m} \hat{d}_{i j} \\
& \Delta R X_{i}(j)=\Delta \bar{X}_{i}(j)+N X_{i}(i) \cdot \hat{d}_{i j}
\end{aligned}
$$

Then $w_{i j}$ can be confirmed in the follow equations.

$$
w_{i j}=\lambda_{i}(j) / \sum_{j=1}^{m} \lambda_{i}(j)
$$

As is shown in (9), the weight vector $w_{i j}$ is direct ratio to relative reliability $\lambda_{i}(j)$, which can evidently increase effect of the neighbor node with higher relative reliability, and enhance the performance of localization and rate of convergence.

\section{In-flight alignment algorithm}

With the Fv-WSN operated on DMDG scheme and the real-time position and velocity supplied by DMDG-3D scheme, the alignment for MINS is turning into a traditional integrated alignment problem, which can apply the technique generated from GPS/INS system.

\subsection{System description and definitions}

In this subsection, we investigate the problem of alignment model for MINS with nonlinear disturbance and uncertain time-relay delay in the output. Consider the following system:

$$
\begin{aligned}
\bar{\Sigma}: \quad & x(k+1)=A_{\lambda} x(k)+B_{\lambda} w(k) \\
& z(k)=H_{\lambda} x(k) \\
& y(k)=C_{\lambda} x(k)+D_{\lambda} w(k) \\
& \hat{y}(k)=r(k) y(k)+(1-r(k)) y(k-1)
\end{aligned}
$$

Here $x(k) \in R^{n}$ is the state vector; $y(k) \in R^{m}$ is the measured output, in Fv-WSN/MINS model, $m=9$,ie., three positon errors ( $\delta L, \delta \lambda, \delta h)$, two horizontal velocity errors $\left(\delta V_{E}, \delta V_{N}\right)$, and four attitude errors $\left(\delta q_{0}, \delta q_{1}, \delta q_{2}, \delta q_{3}\right) ; z(k) \in R^{p}$ is the signal to be estimated; $w(k) \in R^{l}$ is a zero-mean white noise with identity power spectrum density matrix; $r(k) \in R$ is a sequence meeting the statistical regularity of Bernoulli; $A_{\lambda}, B_{\lambda}, C_{\lambda}, D_{\lambda}$ and $H_{\lambda}$ are appropriately dimensioned matrices.

\subsection{Robust $l_{2}-l_{\infty}$ filter algorithm}

We consider a full-order filter for (10) as follows:

$$
\begin{aligned}
& \hat{x}(k+1)=A_{f} \hat{x}(k)+B_{f} \hat{y}(k) \\
& \hat{z}(k)=C_{f} \hat{x}(k)+D_{f} \hat{y}(k)
\end{aligned}
$$

where $\hat{x}(k+1)$ is the filter state, $A_{f}, B_{f}, C_{f}, D_{f}$ are filter parameters with compatible dimensions to be determined.

Then we can obtain the following filtering error system:

$$
\begin{aligned}
& x_{f}(k+1)=A_{c l} x_{f}(k)+A_{d c l} x_{f}(k-1)+B_{c l} \bar{w}(k) \\
& e(k)=C_{c l} x_{f}(k)+C_{d c l} x_{f}(k-1)+D_{c l} \bar{w}(k)
\end{aligned}
$$

where $x(k)=\left[\begin{array}{llllllllll}\delta L & \delta \lambda & \delta h & \delta v_{E} & \delta v_{N} & \delta v_{U} & \delta q_{0} & \delta q_{1} & \delta q_{2} & \delta q_{3}\end{array}\right]^{T}$, and $\bar{w}(k)=\left[\begin{array}{llllll}\varepsilon_{x} & \varepsilon_{y} & \varepsilon_{z} & \nabla_{x} & \nabla_{y} & \nabla_{z}\end{array}\right]^{T}$, the vector $A_{c l}, A_{d c l}, B_{d c}, C_{c l}, C_{d c l}, D_{c l}$ as defined in [4,5].

Theorem 2. Given scalars $\gamma>0$, and filter parameters $A_{f}, B_{f}, C_{f}, D_{f}$.The filtering error system (12) is asymptotically mean-square stable with a prescribed $l_{2}-l_{\infty}$ attenuation leval $\gamma>0$, if there exist $\lambda_{11}, \lambda_{12}, \lambda_{21}, \lambda_{22} \in R^{1}$; positive definite symmetric matrices $P_{1 i}, P_{3 i}, R_{1 i}, R_{3 i}$, and matrices $P_{2 i}, R_{2 i}, V_{1}, V_{2}, V_{3}, \hat{A}_{f}, \hat{B}_{f}, \hat{C}_{f}, D_{f}$ satisfying

$$
\left[\begin{array}{cccccc}
\psi_{11} & \psi_{12} & \psi_{13} & \psi_{14} & \psi_{15} & \psi_{16} \\
* & \psi_{22} & \psi_{23} & \psi_{24} & \psi_{25} & \psi_{26} \\
* & * & -\gamma^{2} I & \psi_{34} & \psi_{35} & \psi_{36} \\
* & * & * & \psi_{44} & 0 & 0 \\
* & * & * & * & \psi_{55} & 0 \\
* & * & * & * & * & -a \psi_{55}
\end{array}\right]<0
$$

where $E=\left[\begin{array}{ll}I_{k * k} & 0_{(n-k) * k}\end{array}\right]^{T}, \psi_{i j}(i, j \leq 6)$ is defined as in [20-25].

Then the filtering error system (12) is mean square exponentially stabilized and has the preset $H_{\infty}$ performance. The filter parameters 
$A_{f}, B_{f}, C_{f}, D_{f}$ in (12) is $A_{f}=V_{2}^{-1} \hat{A}_{f}, B_{f}=V_{2}^{-1} \hat{B}_{f}$, $C_{f}=\hat{C}_{f}, D_{f}=D_{f}$.

When the parameters $\lambda_{11}, \lambda_{12}, \lambda_{21}, \lambda_{22}$ is preset, the formula (13) is simplified as a linear matrix inequality. Then the optimal value of $\gamma$ can be resolved using LMI Toolbox of MATLAB in real time.

\section{Simulation and discussion}

\subsection{Network operation and experiment setup}

The localization procedure begins at the moment of take-off, when the network terminal is starting up and receiving network-assistant information. Then the vehicle climbs up to 1015 meters, and reverts to level flight, $V_{\text {level }}=200 \mathrm{~m} / \mathrm{s}$, till to end of the localization process. Then the vehicle turns into the alignment procedure, which enhances the performance by " $\mathrm{S}$ " maneuver, $T_{\text {alignment }} \leq 40 \mathrm{~s}$. In this section, we use MATLAB 7.1 to test the proposed 3-D localization scheme and in-flight alignment's performance, which are based on the Fv-WSN system and DMDG scheme designed before. The main simulation parameters are listed in Table.1.

Table 1. Main Simulation Parameters

\begin{tabular}{ll}
\hline Parameters & Values \\
\hline Network size $(\mathrm{r}, \mathrm{h})(\mathrm{km})$ & 20,5 \\
The number of sensor nodes & 30 or 50 \\
Distribution of sensor nodes & random \\
MAC/Routing protocol & $\mathrm{DMDG}$ \\
Initial geographic position $(\mathrm{E}, \mathrm{N}, \mathrm{U})\left(^{\circ}\right)$ & $120,30,5$ \\
Initial speed of the vehicle $(\mathrm{E}, \mathrm{N}, \mathrm{U})(\mathrm{m} / \mathrm{s})$ & $0,0,0$ \\
Initial attitude angle of the vehicle $(\varphi, \theta, \gamma)\left({ }^{\circ}\right)$ & $0,0,45$ \\
Vibration of the vehicle $(\mathrm{m} / \mathrm{s})$ & 0.01 \\
Initial misalignment angle of $\mathrm{MINS}(\delta \varphi, \delta \theta, \delta \gamma)$ & $10,1,1$ \\
Constant zero-deviation of accelerometer $(\mathrm{g})$ & $1 \times 10^{-4}$ \\
Constant deviation of gyroscope $\left({ }^{\circ} / \mathrm{h}\right)$ & 0.02 \\
Random zero-deviation of accelerometer $(\mathrm{g})$ & $5 \times 10^{-6}$ \\
Random deviation of gyroscope $\left({ }^{\circ} / \mathrm{h}\right)$ & 0.01 \\
Network time-relay delay $(\tau)(\mathrm{ms})$ & $\leq 50$ \\
\hline
\end{tabular}

\subsection{Simulation result and analysis}

\section{(1) Localization performance of DMDG-3D algorithm}

Fig.2 shows the positional error $\Delta P_{r}$ and coverage rate $P_{\text {cover }}$ in different range measure error $\Delta P_{r}$.

When the distribution of the sensor node is random and the message radius $r_{c}=5000 \mathrm{~m}$, the localization algorithm can guarantee a full coverage in DMDG protocol, while in LEACH protocol the coverage rate had a slight reduction $\left(P_{\text {cover }}=80.32 \%\right.$ when $\left.\Delta P_{r}=40 \%\right)$. Positional error in DMDG protocol is probably $1 / 4$ as in LEACH $\left(\Delta P_{a}=1.3 \%\right.$ when $\left.\Delta P_{r}=5 \%\right)$.

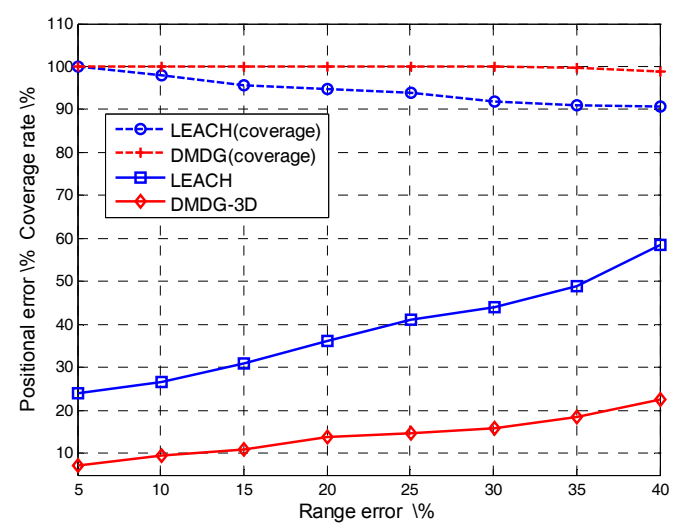

Figure 2. Fv-WSN performance with ranging error

Fig. 3 shows the positional error and RMSE with DMDG-3D algorithm when $r_{c}=5000, \Delta P_{r}=50 \mathrm{~m}$, mean square deviation of environment noise $\sigma=10^{-4}$, and simulated for $500 s$.
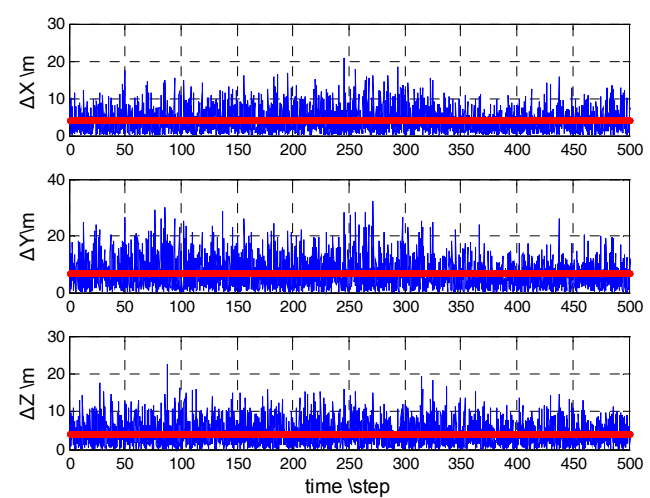

Figure 3. Positional error and RMS error in DMDG-3D The result is listed in Table.2.

Table 2. Analysis of simulation result

\begin{tabular}{cccc}
\hline Parameter & $\Delta P_{\text {rela }} / \%$ & $\Delta P_{\text {abs }} / m$ & $\mathrm{RMSE} / m$ \\
\hline$\Delta x$ & 0.124 & 4.041 & 9.761 \\
$\Delta y$ & 0.263 & 6.682 & 13.254 \\
$\Delta z$ & 0.147 & 4.199 & 10.675 \\
\hline
\end{tabular}

(2) In-flight alignment algorithm with $l_{2}-l_{\infty}$ filter

Fig.4 is the comparation of in-flight alignment performance based on robust $l_{2}-l_{\infty}$ filter and EKF when the time-relay delay $\|\tau\| \leq 50 \mathrm{~ms}$, and other related initial parameters defined as in Table.1. We first seek the optimum value for the variances in 
Theorem. 1 with LMI toolbox, obtain $\gamma_{\min }=0.46$, then design the robust $l_{2}-l_{\infty}$ filter (11). Simulation results are shown in Fig.4 and detailed performances are listed in Table.3.

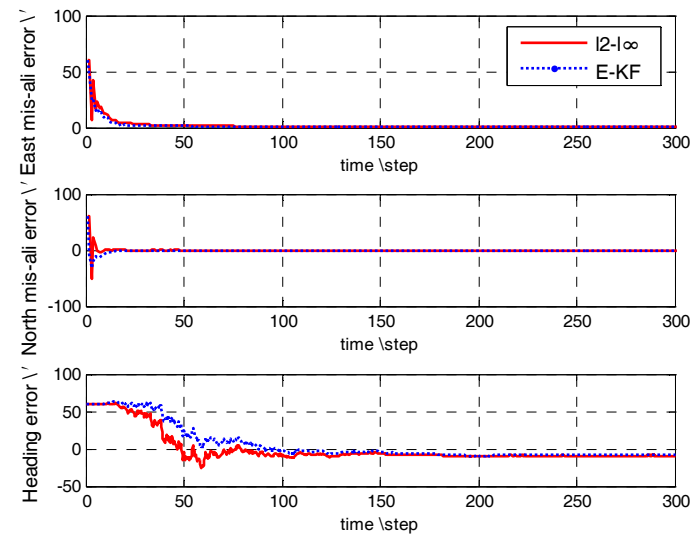

Figure 4 (a). Comparation of misalignment errors between robust $l_{2}-l_{\infty}$ filter and EKF
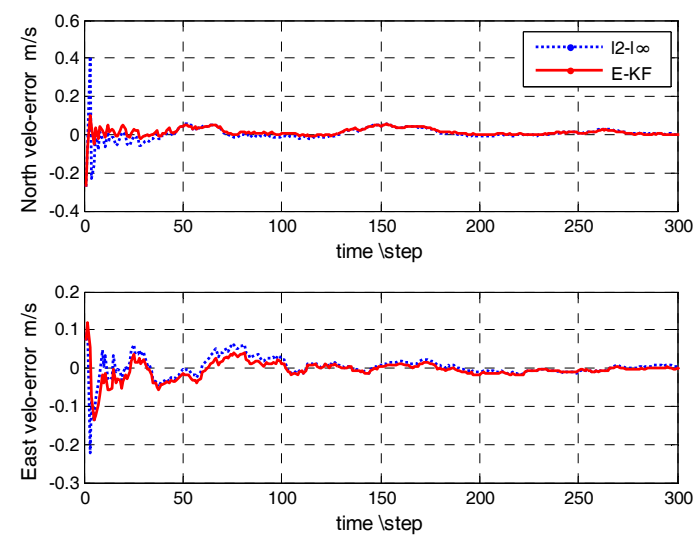

Figure 4 (b). Comparation of velocity errors between robust $l_{2}-l_{\infty}$ filter and EKF

Table 3. Analysis of in-flight alignment simulation result based on Fv-WSN

\begin{tabular}{lccccc}
\hline Parameter & $\Delta \theta /^{\prime \prime}$ & $\Delta \gamma /{ }^{\prime \prime}$ & $\Delta \psi /^{\prime}$ & $\Delta V_{\mathrm{E}}$ & $\Delta V_{\mathrm{N}}$ \\
\hline$E K F$ & 33.76 & 30.15 & 5.12 & 0.023 & 0.035 \\
$12-l \infty$ & 21.14 & 21.01 & 3.54 & 0.012 & 0.022 \\
\hline
\end{tabular}

\section{Conclusion and future directions}

In this paper we propose a new 3D localization scheme and an in-flight alignment algorithm for aircrafts in fleet network, which is established based on WSN technique and with a DMDG networking scheme. Simulation results show that the weighting localization algorithm can guarantee an accurate position estimation and high coverage in a sparse dynamic network, and the $l_{2}-l_{\infty}$ filter can acquire a more rapid and precise calculating results with random time-relay delay. To further improve the accuracy of the localization and alignment, more work should be taken to analyze more complex error including NLOS, and modified topology control arithmetic for load-balance, which will be discussed in our future paper.

\section{Acknowledgments}

This work is supported by the National Defense Previous Research Foundation of China (Grant No. 51309060401). The authors express sincerely appreciation to the reviewers of this paper for their helpful recommendations.

\section{References}

[1] Kwangjin Kim, Chan Gook Park. "In-flight alignment algorithm based on non-symmetric unscented transformation". SICE-ICASE international joint conference 2006, October, 2006, pp. 4916-4920.

[2] Costa J A, Patwari N, Hero A O. "Distributed weighted-multidimensional scaling for node localization in sensor networks". ACM Transactions on Sensor Networks, 2006, 2(1), pp. 39-64.

[3] Jamshaid Ali, Muhammad Ushaq. "A consistent and robust Kalman filter design for in-motion alignment of inertial navigation system". Measurement. October, 2008.

[4] WANG Wu, YANG Fu-Wen. "Robust $H_{\infty}$ filtering for networked uncertain systems with random time delays". Acta automatica sinica, 2007, 33(5), pp. 557-560.

[5] L Q Zhang, X B Zhou, Q Cheng. "Landscape-3D: A robust localization scheme for sensor networks over complex 3D terrains". In Proc.31st IEEE conference, 2006, pp. 239-246..

[6] Chao Gao, Guorong Zhao, Shuang Pan. "Distributed Multi-weight Data-gathering and Aggregation Protocol in Fleet Wireless Sensor Networks: Optimal and Heuristic Algorithms". International Journal of Intelligent Engineering and Systems. Vol.2, No.4, 2009: 1-8.

[7] Chao Gao, Guorong Zhao, Shuang Pan, et al. "DMDG : A Distributed Data-aggregation and Routing Protocol for Fleet Wireless Sensor Networks". 2009 Second International Conference on Intellegent Networks and Intelligent Systems. 2009: 150-155.

[8] Hui Qu, Stephen B. Wicker. "Co-designed anchor-free localization and location-based routing algorithm for rapidly-deployed wireless sensor networks". Information fusion. 2008(9): 425-439. 
[9] Yibin Yu, Xiaoning Xue, XiaoZhen Wang. "Location Discovery for Three-Dimensional Sensor-Actor Networks Using Alternating Combination Quadrilateration". $20066^{\text {th }}$ Internation Conference on ITS Telecommunications Proceedings. 2006: 1021-1024.

[10]Dai Gui-lan, Zhao Chong-chong, et al. "A localization Scheme based on sphere for wireless sensor network in 3D". Acta electronica sinica, 2008, 36(7): 1297-1303.

[11]Dario Pompili, Tommaso Melodia, Ian F. Akyildiz. "Three-dimensional and two-dimensional deployment analysis for underwater acoustic sensor networks". Ad Hoc Networks. 2009(7): 778-790.

[12]K.Akkaya, S.Janapala. "Maximizing connected coverage via controlled actor relocation in wireless sensor and actor networks". Computer Networks. 2008(52):2779-2796.

[13]Horacio A.B.F. Oliveira, Azzedine Boukerche, Eduardo F. Nakamura, et al. "Localization in time and space for wireless sensor networks: An efficient and lightweight algorithm". Performance Evaluation. 2009(66): 209-222.

[14]Alireza A. Nezhad, Ali Miri, Dimitris Makrakis. "Location privacy and anonymity preserving routing for wireless sensor networks". Computer Networks. 2008(52): 3433-3452.

[15]Jang-Sub Kim, Jaehan Lee, Erchin Serpedin, et al. “A robust estimation scheme for clock phase offsets in wireless sensor networks in the presence of non-Gaussian random delays". Signal Processing. 2009(89): 1155-1161.

[16]Erfu Yang, Ahmet T. Erdogan, Tughrul Arslan, etal. "Adaptive Formation Control and Bio-inspired Optimization of a Cluster-based Satellite Wireless Sensor Network". NASA/ESA Conference on Adaptive Hardware and Systems. 2008.06: 432-439.

[17]Dawood Al-Abri, Janise McNair. "On the interaction between localization and location verification for wireless sensor networks". Computer Networks. 2008(52): 2713-2727.

[18]Dandan Liu, Xiaohua Jia, Ivan Stojmenovic. "Quorum and connected dominating sets based location service in wireless ad hoc, sensor and actuator networks". Computer Communications. 2007(30): 3627-3643.

[19]Neal Patwari, Joshua N.Ash, Spyros Kyperountas, et al. "Locating the nodes: Cooperative localization in wireless sensor networks". IEEE Signal Processing Magazine. 2005.07: 54-69.

[20]Wang Wu, Yang Fu-Wen. "Robust $H_{\infty}$ filtering for networked uncertain systems with random time delays". Acta automatica sinica, 2007,33(5): 557-560.

[21]Hamid Reza Karimi, Huijun Gao. "Mixed $H_{2} / H_{1}$ output-feedback control of second-order neutral systems with time-varying state and input delays". ISA Transactions, 2008 (47): 311-324.

[22]Hongli Dong, Zidong Wang, Huijun Gao.
" $H_{\infty}$ filtering for systems with repeated scalar nonlinearities under unreliable communication links". Signal Processing, 2009.02: 1-9.

[23]James Lam, Huijun Gao, Changhong Wang. "Stability analysis for continuous systems with two additive time-varying delay components". Systems \& Control Letters, 2007(56): 16-24.

[24]Lu Ren-quan, Wang Jun-hong, Xue An-ke et al. "Robust $H_{\infty}$ filtering for a class of uncertain lurie time-delay singular systems". Acta Automatica Sinica. 2007, 33(3): 292-296.

[25]Wen-Hwa Liao, Kuei-Ping Shih, Yu-Chee Lee. "A localization protocol with adaptive power control in wireless sensor networks". Computer Communications. 2008(31): 2496-2504. 